\title{
Is there any advantage from continuation of trastuzumab beyond progression in metastatic her positive gastric cancer? report of two cases and literature review
}

\author{
Rachid Tanz ${ }^{1,2 *}$, Tarik Mahfoud ${ }^{1}$, Ilyas El Alami' ${ }^{1}$, Aziz Bazine ${ }^{1}$, Hassan Errihani $^{3}$ and Mohamed Ichou ${ }^{1}$ \\ ${ }^{1}$ Medical Oncology Department, Military Hospital Mohamed V, Rabat, Morocco \\ ${ }^{2}$ Faculty of Medicine and Pharmacy, Rabat, Morocco \\ ${ }^{3}$ National Institute of Medical Oncology, Rabat, Morocco
}

\begin{abstract} progressive disease to first line treatment containing Trastuzumab. safe, practical and may improve chance for survival.

\section{Introduction}

In metastatic patients with HER2 overexpression the addition of Trastuzumab to first line chemotherapy improves tumor response and chance for survival. The optimal duration of Trastuzumab in this setting still unknown as well as the benefit of Trastuzumab continuation beyond progression in advanced gastric cancer. We report two patients who favorably responded to second line chemotherapy with Trastuzumab continuation following progressive disease to first line treatment containing Trastuzumab. As seen in our cases and according to published data the continuation of Trastuzumab beyond progressive disease in metastatic HER positive gastric cancer is safe, practical and may improve chance for survival.
\end{abstract}

Background: Gastric cancer is a widespread cancer and one of the leading causes of cancer death worldwide. In metastatic patients with HER2 overexpression the addition of Trastuzumab to first line chemotherapy improves tumor response and chance for survival. The optimal duration of Trastuzumab in this setting still unknown as well as the benefit of Trastuzumab continuation beyond progression in advanced gastric cancer.

Case presentation: We report two Moroccan female patients who favorably responded to second line chemotherapy with Trastuzumab continuation following

Conclusion: Our report and the review of literature showed that Trastuzumab continuation beyond progressive disease in metastatic HER positive gastric cancer is

\section{Case 1}

A 46-years-old Moroccan female diagnosed with HER2 positive advanced gastric adenocarcinoma and metastasis to liver, lymph nodes, ovary and lungs was admitted for care to our institution. Due to a disease relapse after an initial successful response to a combination of Oxaliplatin $+5 \mathrm{FU}$ and Trastuzumab she received a second line chemotherapy that consists of Trastuzumab combined with FOLFIRI regimen (Irinotecan plus 5-FU/LV). The patient showed a favorable and prolonged response to this protocol. In addition; the treatment was well tolerated without remarkable side effects (Figures 1 and 2).

\section{Case 2}

A 53-years-old Moroccan female was diagnosed for a gastric adenocarcinoma with overexpression of HER2. CT scan showed a gastric tumor with liver metastasis and lymph nodes metastases. She received six cycles of first line chemotherapy (FOLFOX+Trastuzumab) with partial response. Six months after treatment interruption due to the patient lassitude, she experienced disease progression (Figure 3 ). The patient started systemic chemotherapy with FOLFIRI + Trastuzumab. After six cycles restaging CT (Figure 4) showed a marked decrease in the size of the primary gastric mass and complete resolution of hepatic metastasis. Clinically, the patient was feeling remarkably better. No cardiological adverse events were noted.

\section{Discussion}

Gastric cancer is one of the most frequent and fatal digestive cancers with approximately 72,300 deaths in 2012 [1]. In metastatic or locally advanced disease chemotherapy remains standard of care. Several chemotherapy treatments are currently approved in management of metastatic gastric cancer in first line [2]. There is not a clear superiority between protocols but the association of a fluoropyrimidine and platinum sometimes associated with docetaxel or epirubicin appears to be the most prescribed treatment in first line [3]. Despite the use of poly-chemotherapy prognostic still poor in metastatic setting hence the necessity to develop other combinations. There is a category of tumors in gastric cancer that harbors an overexpression of HER2 [4]. The incidence of this overexpression is variable (from 8.2 to $53.4 \%$ )

*Correspondence to: Rachid Tanz, Medical Oncology Department, Military Hospital Mohamed V, Rabat, Morocco, Tel: 00212666042442; Email: tanz rachid@gmail.com

Key words: advanced gastric cancer, HER2 overexpression, trastuzumab continuation

Received: February 21, 2018; Accepted: April 17, 2018; Published: April 20, 2018 


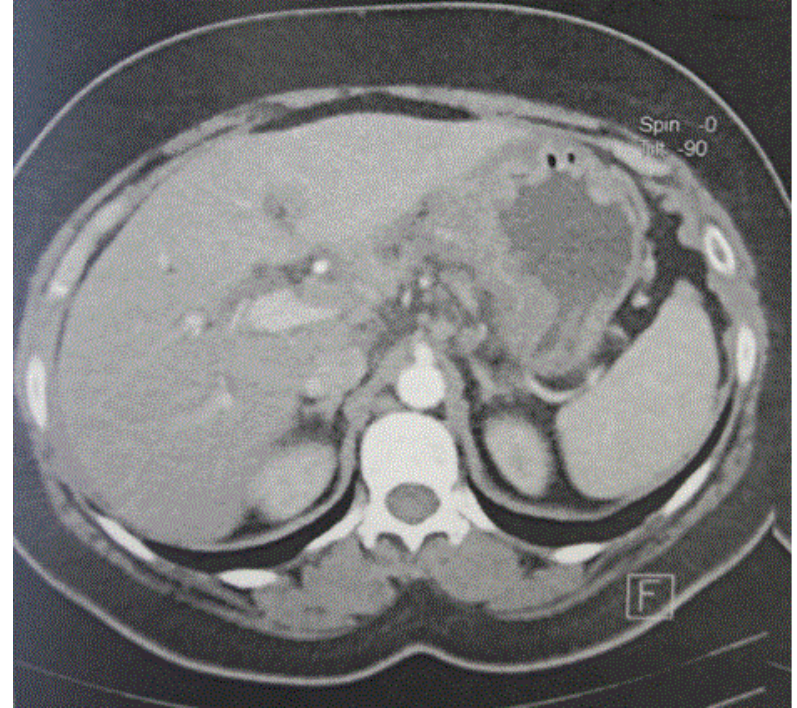

Figure 1. Transverse CT image taken from case 1 before treatment: This image shows advanced gastric tumor with metastasis to liver and nodes.a

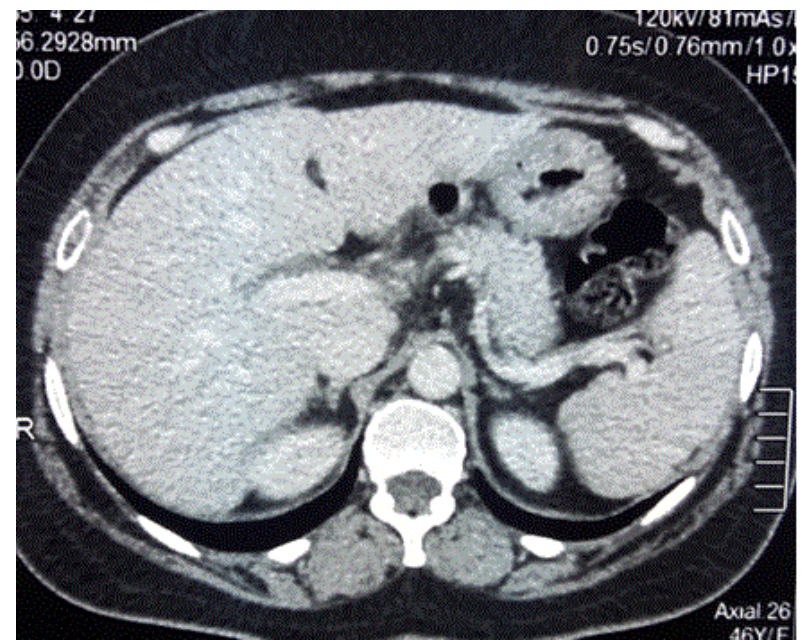

Figure 2. Transverse CT image taken from Case 1 after six cycles of FOLFIRI+Trastuzumab: This image shows a marked decrease in the size of the primary gastric mass and complete resolution of the lymph nodes metastasis.

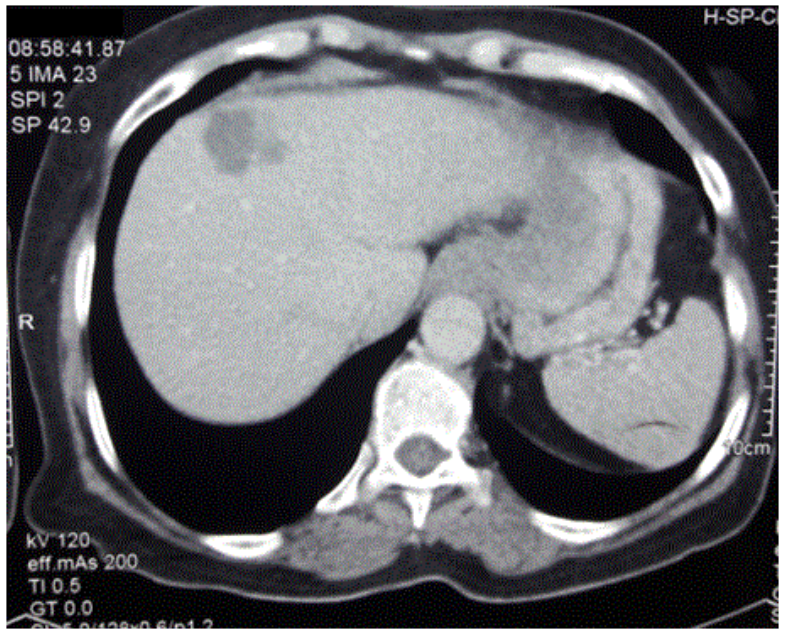

Figure 3. Transverse CT image taken from case 2 before treatment: This image shows advanced gastric tumor with metastasis to liver and nodes.

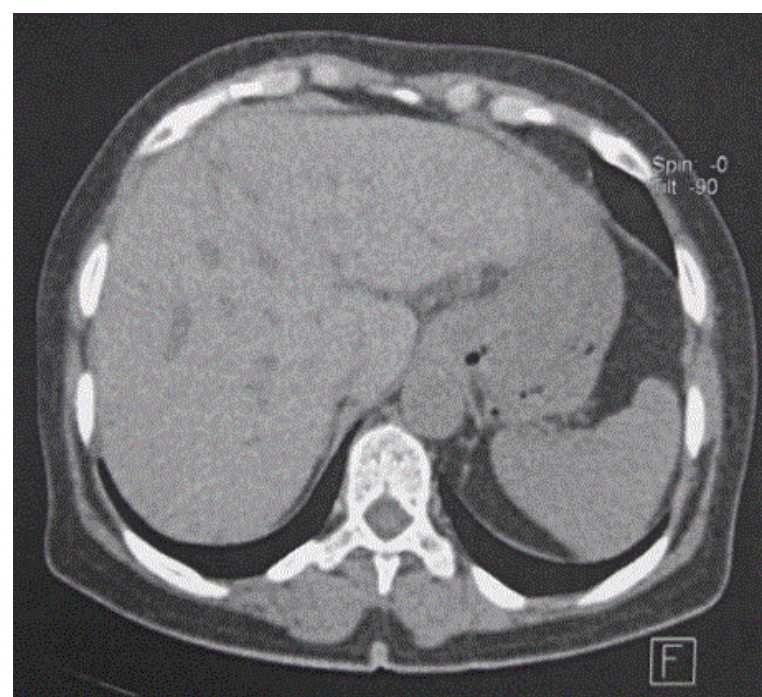

Figure 4. Transverse CT image taken from Case 2 after six cycles of FOLFOX+HERCEPTIN: This image shows a marked decrease in the size of the primary gastric mass and complete resolution of hepatic metastasis.

between publications [5]. For patients with overexpression of HER2, the addition of Trastuzumab to standard chemotherapy provided significant improvement in response rate, progression free survival and overall survival compared to chemotherapy alone in a randomized phase 3 trial [6]. Despite this real positive impact of the use of Trastuzumab in metastatic HER2 positive gastric cancer in first line the benefit of its continuation beyond progression still unknown. Evidence of benefice of Trastuzumab in second line in Trastuzumab-naïve patients comes from a phase II study published in 2013. In this trial Trastuzumab combined to Paclitaxel in a population of metastatic HER positive metastatic gastric cancer patients provided an overall response rate of $37.2 \%$ and median of progression free survival of $5.2 \mathrm{mo}$ [7].

Even in third line the use of the combination of Trastuzumab and FOLFIRI provided disease complete response in the case reported by Weissinger, et al. [8]. The reintroduction of Trastuzumab with FOLFIRI permitted a prolonged response in the case of a patient who had progressed few months after completion of the first line Trastuzumab based chemotherapy [9]. In a retrospective analysis published in 2014, Trastuzumab was used with a new chemotherapy protocol after failure of a first line Trastuzumab based treatment was reported in four patients. In this study the continuation of Trastuzumab leads to a significant response with prolonged remeTdial in two cases [10]. A retrospective study recently published reporting the experience of the MD Anderson Cancer Center. In this study Trastuzumab was continued beyond progression in combination with Irinotecan and 5-FU in twenty-five patients. Progression free survival was 5 months for those patients without significant side events due to Trastuzumab. The authors concluded that the continuation of Trastuzumab beyond progression is safe and practicable [11].

More recently controversial results were reported in a phase $2 / 3$ study [12]. The Gatsby-trial was a multicenter, randomized study designed to evaluate the efficacy and safety of ado-trastuzumab emtansine compared to standard treatment with docetaxel or paclitaxel in patients with HER2-positive advanced gastric cancer. Eligible patients had HER2-positive advanced gastric cancer and progressed during or after first-line therapy. Patients were randomly assigned to receive intravenous trastuzumab emtansine or physician's choice of a taxane. In this trial Trastuzumab emtansine was not superior to taxane 

review

in patients with previously treated, HER2-positive advanced gastric cancer. And authors concluded that there is still an unmet need in this patient group and therapeutic options remain limited.

\section{Conclusion}

Our report and the clinical survey showed that Trastuzumab continuation beyond progressive disease in metastatic HER positive gastric cancer is safe, possible and may improve chance for survival. This approach need to be evaluated in more powerful study.

\section{Consent to publish}

All patients gave their consent for participation in this study and for the publication

Written informed consent was obtained from the patients for publication of this case report and any accompanying images. A copy of the written consent is available for review by the Editor-in-Chief of this journal

\section{Ethics}

This study was approved by the committee of ethics of military hospital of Rabat

\section{Competing interests}

All authors declare not have any competing interests in the manuscript.

\section{Authors' contributions}

All authors had equally contributed to this work

\section{Availability of data and material}

Data sharing not applicable to this article as no datasets were generated or analyzed during the current study.

\section{References}

1. Torre LA, Bray F, Siegel RL, Ferlay J, Lortet-Tieulent J, et al. (2015) Global cancer statistics, 2012. CA Cancer J Clin 65: 87-108. [Crossref]
2. Waters JS, Norman A, Cunningham D, Scarffe JH, Webb A, et al. (1999) Long-term survival after epirubicin, cisplatin and fluorouracil for gastric cancer: results of a randomized trial. Br J Cancer 80: 269-272. [Crossref]

3. Al-Batran SE, Hartmann JT, Probst S, Schmalenberg H, Hollerbach S, et al. (2008) Phase III trial in metastatic gastroesophageal adenocarcinoma with fluorouracil, leucovorin plus either oxaliplatin or cisplatin: a study of the Arbeitsgemeinschaft Internistische Onkologie. J Clin Oncol 26: 1435-1442. [Crossref]

4. Hofmann M, Stoss O, Shi D, Büttner R, van de Vijver M, et al. (2008) Assessment of a HER2 scoring system for gastric cancer: results from a validation study. Histopathology 52: 797-805.

5. Jørgensen JT (2010) Targeted HER2 treatment in advanced gastric cancer. Oncology 78: 26-33. [Crossref]

6. Bang YJ, Van Cutsem E, Feyereislova A, Chung HC, Shen L, et al. (2010) Trastuzumab in combination with chemotherapy versus chemotherapy alone for treatment of HER2positive advanced gastric or gastro-oesophageal junction cancer (ToGA): a phase 3, open-label, randomised controlled trial. Lancet 376: 687-697. [Crossref]

7. Nishikawa K, Takahashi T, Takaishi H, Miki A, Noshiro H, et al. (2017) Phase II study of the effectiveness and safety of trastuzumab and paclitaxel for taxane- and trastuzumab-naïve patients with HER2-positive, previously treated, advanced, or recurrent gastric cancer (JFMC45-1102). Int J Cancer 140: 188-196. [Crossref]

8. Weissinger F, Reymond M, Dumke K, Krüger M. (2011) Successful treatment of a patient with HER2-positive metastatic gastric cancer with third-line combination therapy with Irinotecan, 5-Fluorouracil, Leucovorin and Trastuzumab (FOLFIRI-T) Onkologie 34: 548-551.

9. Dubreuil O, Zaanan A, Pellerin O, Bachet JB, Rougier P, et al. (2015) Trastuzumab re-introduction with FOLFIRI for treatment of HER2 overexpression- advanced gastric adenocarcinoma following failure of other trastuzumab-based chemotherapy regimens. CRCM 4: 131-136.

10. Elamin YY, Rafee S, Nur MM, Osman N, Reynolds JV, et al. (2014) HER2 positive gastric and gastroesophageal adenocarcinoma: An Irish tertiary center experience. Int J Cancer Clin Res 1: 008.

11. Al-Shamsi HO, Fahmawi Y, Dahbour I, Tabash A, Rogers JE, et al. (2016) Continuation of trastuzumab beyond disease progression in HER2-positive metastatic gastric cancer: the MD Anderson experience. J Gastrointest Oncol 7: 499-505. [Crossref]

12. Thuss-Patience PC, Shah MA, Ohtsu A, Van Cutsem E, Ajani JA, et al. (2017) Trastuzumab emtansine versus taxane use for previously treated HER2-positive locally advanced or metastatic gastric or gastro-oesophageal junction adenocarcinoma (GATSBY): An international randomised, open-label, adaptive, phase 2/3 study. Lancet Oncol 18: 640-653. 